\title{
Bayesian Prediction of the Overhaul Effect on a Repairable System with Bounded Failure Intensity
}

\author{
Preeti Wanti Srivastava and Nidhi Jain \\ Department of Operational Research, University of Delhi, Delhi 7, India \\ Correspondence should be addressed to Preeti Wanti Srivastava,pw_srivastava@yahoo.co.in
}

Received 13 December 2010; Revised 20 June 2011; Accepted 21 June 2011

Academic Editor: Ratna Babu Chinnam

Copyright ( $) 2011$ P. W. Srivastava and N. Jain. This is an open access article distributed under the Creative Commons Attribution License, which permits unrestricted use, distribution, and reproduction in any medium, provided the original work is properly cited.

\begin{abstract}
This paper deals with the Bayes prediction of the future failures of a deteriorating repairable mechanical system subject to minimal repairs and periodic overhauls. To model the effect of overhauls on the reliability of the system a proportional age reduction model is assumed and the 2-parameter Engelhardt-Bain process (2-EBP) is used to model the failure process between two successive overhauls. 2-EBP has an advantage over Power Law Process (PLP) models. It is found that the failure intensity of deteriorating repairable systems attains a finite bound when repeated minimal repair actions are combined with some overhauls. If such a data is analyzed through models with unbounded increasing failure intensity, such as the PLP, then pessimistic estimates of the system reliability will arise and incorrect preventive maintenance policy may be defined. On the basis of the observed data and of a number of suitable prior densities reflecting varied degrees of belief on the failure/repair process and effectiveness of overhauls, the prediction of the future failure times and the number of failures in a future time interval is found. Finally, a numerical application is used to illustrate the advantages from overhauls and sensitivity analysis of the improvement parameter carried out.
\end{abstract}

\section{Introduction}

A repairable system is a system that, after failing to perform one or more of its functions satisfactorily, can be restored to satisfactory performance.

Most repairable mechanical systems are subjected to degradation phenomena with operating time, so that the failures become increasingly frequent with time. Such systems often undergo a maintenance policy. Maintenance extends system's lifetime or at least the mean time to failure, and an effective maintenance policy can reduce the frequency of failures and the undesirable consequences of such failures. Maintenance can be categorized into two classes: corrective and preventive actions. Corrective maintenance, called repair, is all actions performed to restore the system to functioning condition when it fails. Preventive maintenance is all actions performed to prevent failures when the system is operating. Corrective and preventive maintenance actions are generally classified in terms of their effect on the operating conditions of the system. Pham and Wang [1] classified them as perfect maintenance, minimal maintenance, imper- fect maintenance, and worse maintenance. At one extreme is the assumption of perfect maintenance, that a system is restored to good-as-new condition after maintenance. At the other extreme is the bad-as-old assumption that the failure rate of a system is not enhanced by maintenance. In real-world situations, maintenance generally enhances the condition of the equipment at a level between these two extremes, that is, imperfect maintenance.

We consider a system that deteriorates with age and receives two kinds of maintenance actions: minimal repair and overhaul. When a failure occurs, minimal repair is carried out. The minimal repair is a corrective maintenance action that brings the repaired equipment to the conditions it was just before the failure occurrence (badas-old). Hence, the reliability of the system decreases with operating time until it reaches unacceptable values. When it reaches unacceptable values or at prefixed epochs, preventive maintenance action (overhaul) is performed so as to improve the system condition and hence reduce the probability of failure occurrence in the following interval. However, overhaul cannot return the system to "good-as-new", and 
thus it can be treated as imperfect repair. When the overhaul is effective, the reliability of the system improves significantly. An overhaul usually consists of a set of preventive maintenance actions such as oil change, cleaning, greasing, and replacing some worn components of the system.

Many imperfect repair models have already been proposed [1]. Malik [2] proposed a general approach to model the improvement effect of maintenance, where each maintenance reduces the age of the unit in the view of the rate of occurrences of failures. Each maintenance is assumed to reduce proportionally the operating time elapsed from the previous maintenance. Malik's proportional age reduction (PAR) model for imperfect maintenance is a generalization of good-as-new and bad-as-old. On the basis of this general model, Shin et al. [3] have proposed a PAR model which assumes that each major overhaul reduces proportionally the age of the equipment by a fraction of the epoch of the overhaul. Shin's paper proposes a method of data analysis to estimate the parameters of the failure process and the maintenance effect for a repairable unit. Two parametric families of intensity functions are considered, power law [4-11] and log linear [12]. To model different effects of rejuvenation of the preventive maintenance, two classes of models have been proposed by Jack [13].

In contrast to the classical approach used by them, Bayes approach has been used by several authors as it helps in incorporating prior information and/or technical knowledge on the failure mechanism and on the overhaul effectiveness into the inferential procedure. Pulcini [14] deals with the statistical analysis, from a Bayes viewpoint, of the failure data of repairable mechanical units subjected to minimal repairs and periodic overhauls. The effect of overhauls on the reliability of the system is modeled by PAR model and the power law process (PLP) is used to model the failure process between two successive overhauls. Pulcini provided Bayes point and interval estimation of model parameters (and functions thereof), as well as testing procedures on the effectiveness of the performed overhauls. Further, Pulcini [15] deals with the prediction, from a Bayes viewpoint, of future failures of repairable mechanical units subjected to minimal repairs and periodic overhauls. In [15], also PLP is used to model the failure process between two successive overhaul epochs, and the effect of overhauls on the reliability of the system is modeled by proportional age reduction model.

In PLP models, the increasing failure intensity tends to infinity as the system age increases. However, it is noted that the failure intensity of deteriorating repairable systems attains a finite bound when beginning from a given system age, repeated minimal repair actions are combined with some overhauls performed in order to oppose the growth of failure intensity with the operating time. The average behavior of the intensity function due to the consecutive steps with increasing intensity between two subsequent overhauls results in globally constant asymptotic intensity. If such data is analyzed through models with unbounded increasing failure intensity, such as the PLP, then pessimistic estimates of the system reliability will arise, and incorrect preventive maintenance policy may be defined.
Engelhardt and Bain [16] were the first to suggest NHPP with an increasing bounded intensity, called Engelhardt-Bain process (EBP). But the mathematical simplicity of their 1parameter model makes it inadequate to analyze failure data in many cases. Most recently, Pulcini [17] proposed a 2parameter NHPP called the bounded intensity process (BIP) to fit the failure data of deteriorating repairable systems showing bounded intensity function. Attardi and Pulcini [18] proposed a new bounded intensity process called 2parameter Engelhardt-Bain process (2-EBP) whose failure intensity function is given by

$$
\lambda(t)=\eta\left(\frac{t}{t+\theta}\right), \quad \eta, \theta>0, t \geq 0
$$

The failure intensity in (1) tends to its asymptote more slowly than the BIP intensity and is in a way a compromise between the PLP and BIP models. When $\eta \equiv 1 / \theta$, the intensity in (1) degenerates into the EBP intensity.

This paper deals with the Bayes prediction of the future failures of a deteriorating repairable mechanical system subjected to minimal repairs and periodic overhauls. To model the effect of overhauls on the reliability of the system, a proportional age reduction model is assumed, and the 2-parameter Engelhardt-Bain process (2-EBP) is used to model the failure process between two successive overhauls. On the basis of the observed data and of a number of suitable prior densities reflecting varied degrees of belief on the failure/repair process and effectiveness of overhauls, the prediction of the future failure times and the number of failures in a future time interval is found. Finally, a numerical application is used to illustrate the advantages of overhauls.

\section{Basic Assumptions}

(i) Failure rate of the system is an increasing function of time that attains a finite bound as $t$ tends to infinity.

(ii) System is subjected to two kinds of maintenance actions: minimal repair and overhaul.

(iii) The times to perform maintenance actions are ignored.

(iv) Minimal repair will restore the failure rate only to bad-as-old condition. But overhauls will improve the system to a condition between bad-as-old and goodas-new.

(v) The failure density function is not changed by overhauls.

(vi) The quality of an overhaul is dependent on improvement factor $\rho(0 \leq \rho \leq 1)$.

(vii) The improvement parameter $\rho$ has a uniform value over all the overhaul actions.

(viii) The $j$ th overhaul reduces the age $t$ of the system by a fraction $\rho$ of the epoch $x_{j}$ of the overhaul.

(ix) The effect of overhauls on the reliability of the system is modeled by proportional age reduction model, and the 2-parameter Engelhardt-Bain process (2-EBP) 
is used to model the failure process between two successive overhauls, say $\left(x_{j}, x_{j+1}\right)$.

(x) $x_{1}, x_{2}, \ldots, x_{k}$ are the $k$ overhaul epochs, which may coincide with failure times.

\section{Model Formulation}

Let $t_{1}<t_{2}<\cdots<t_{n}$ denote the $n$ failure times of the repairable system observed till $T$. If $T \equiv t_{n}$, that is, the process is observed till $n$th failure, then it is failure-truncated sampling, and $T$ is a random variable. If $T>t_{n}$, then it is time-truncated sampling, where $T$ is a prefixed quantity, and $n$ is a random variable.

The 2-EBP model is an NHPP whose failure intensity is of the form

$$
\lambda(t)=\eta\left(\frac{t}{t+\theta}\right), \quad \eta, \theta>0, t \geq 0,
$$

which is an increasing bounded intensity function with the operating time $t$, equal to 0 at $t=0$ and approaching an asymptote of $\eta$ as $t$ increases. So, the parameter $\eta$ is the asymptotic value of the intensity function: $\eta \equiv \lambda_{\infty}$, and the parameter $\theta$ is a measure of the initial increasing rate of $\lambda(t)$ : the smaller $\theta$ is, the faster the failure intensity increases until it approaches $\eta$.

Thus, the initial failure intensity, that is, the intensity function till the first overhaul epoch $x_{1}$, is

$$
\lambda_{1}(t)=\eta\left(\frac{t}{t+\theta}\right), \quad t \leq x_{1}
$$

The conditional intensity function at a generic time $t$ in the interval $\left(x_{j}, x_{j+1}\right)$, given $x_{j}$ is,

$$
\begin{aligned}
\lambda_{j+1}\left(t \mid x_{j}\right) & =\lambda_{1}\left(t-\rho x_{j}\right) \\
& =\eta\left(\frac{t-\rho x_{j}}{t-\rho x_{j}+\theta}\right) ; \quad x_{j}<t \leq x_{j+1} .
\end{aligned}
$$

The expected number of failures between two successive overhaul epochs is

$$
\begin{aligned}
E\left\{N\left(x_{j}, x_{j+1}\right)\right\} & =\int_{x_{j}}^{x_{j+1}} \lambda_{j+1}\left(t \mid x_{j}\right) d t \\
& =\eta\left[\left(x_{j+1}-x_{j}\right)-\theta \log \left(\frac{x_{j+1}-\rho x_{j}+\theta}{x_{j}-\rho x_{j}+\theta}\right)\right] .
\end{aligned}
$$

The cumulative value of expected number of failures from 0 up to a generic time $\tau$ in the interval $\left(x_{i}, x_{i+1}\right)$ is

$$
\begin{aligned}
N_{(\tau)}= & {\left[\sum_{j=0}^{i-1} E\left\{N\left(x_{j}, x_{j+1}\right)\right\}\right] } \\
& +E\left\{N\left(x_{i}, x_{\tau}\right)\right\}, \quad x_{i} \leq \tau \leq x_{i+1}, x_{0}=0
\end{aligned}
$$

$$
\begin{aligned}
& =\eta\left[\sum_{j=0}^{i-1}\left(\left(x_{j+1}-x_{j}\right)-\theta \log \left(\frac{x_{j+1}-\rho x_{j}+\theta}{x_{j}-\rho x_{j}+\theta}\right)\right)\right] \\
& +\eta\left[\left(\tau-x_{i}\right)-\theta \log \left(\frac{\tau-\rho x_{i}+\theta}{x_{i}-\rho x_{i}+\theta}\right)\right] .
\end{aligned}
$$

\section{Likelihood Function}

The likelihood function based on observed data: $t_{1}, t_{2}, \ldots$, $t_{n}, T$, is

The conditional probability that the unit of age $t_{i-1}$ will fail before $t_{i}$ is

$$
\begin{aligned}
F\left(t_{i} \mid t_{i-1}\right) & =P\left[T \leq t_{i} \mid T>t_{i-1}\right] \\
& =\frac{F\left(t_{i}\right)-F\left(t_{i-1}\right)}{\bar{F}\left(t_{i-1}\right)} \\
& =\frac{-\left(1-F\left(t_{i}\right)\right)+\left(1-F\left(t_{i-1}\right)\right)}{\bar{F}\left(t_{i-1}\right)} \\
& =\frac{\bar{F}\left(t_{i-1}\right)-\bar{F}\left(t_{i}\right)}{\bar{F}\left(t_{i-1}\right)} \\
& =1-\frac{\bar{F}\left(t_{i}\right)}{\bar{F}\left(t_{i-1}\right)} .
\end{aligned}
$$

The conditional reliability just before the $i$ th failure will be

$$
\begin{aligned}
\bar{F}\left(t_{i} \mid t_{i-1}\right) & =1-F\left(t_{i} \mid t_{i-1}\right) \\
& =\frac{\bar{F}\left(t_{i}\right)}{\bar{F}\left(t_{i-1}\right)} \\
& =e^{-\int_{t_{i-1}}^{t_{i}} r(u) d u},
\end{aligned}
$$

and the conditional pdf is $f\left(t_{i} \mid t_{i-1}\right)$.

If the data is time truncated, the joint pdf of the failure times $\left(t_{1}, t_{2}, \ldots, t_{n}\right)$ is

$$
\begin{aligned}
f\left(t_{1}, t_{2}, \ldots, t_{n}\right)= & f\left(t_{1}\right) f\left(t_{2} \mid t_{1}\right) \cdots f\left(t_{n} \mid t_{1}, t_{2}, \ldots, t_{n-1}\right) \\
& \times \bar{F}\left(T \mid t_{n}\right) \\
= & \lambda\left(t_{1}\right) \cdot e^{-\int_{0}^{t_{1}} \lambda(u) d u} \cdot \lambda\left(t_{2}\right) \\
& \cdot e^{\left.-\iint_{0}^{t_{2}} \lambda(u) d u-\int_{0}^{t_{1}} \lambda(u) d u\right]} \cdots \lambda\left(t_{n}\right) \\
& \cdot e^{-\left[\int_{0}^{t_{n}} \lambda(u) d u-\int_{0}^{t_{n-1}} \lambda(u) d u\right]} \\
& \cdot e^{-\int_{t_{n}}^{T} \lambda(u) d u} \\
= & {\left[\prod_{i=1}^{n} \lambda\left(t_{i}\right)\right] \cdot e^{-\int_{0}^{T} \lambda(u) d u}, }
\end{aligned}
$$

where $T$ is the truncation time.

As $x_{1}, x_{2}, \ldots, x_{k}$ are the $k$ overhaul epochs, they provide disjoint fixed intervals $\left(x_{0}, x_{1}\right),\left(x_{1}, x_{2}\right), \ldots,\left(x_{k-1}, x_{k}\right)$. Since 
the overhaul epochs are prescheduled, they are not random. Let the time interval $\left(x_{j}, x_{j+1}\right)$ be the $(j+1)$ th period.

From (4) and (9a), for $t \in\left(x_{j}, x_{j+1}\right)$, the joint pdf of failure times in $\left(x_{j}, x_{j+1}\right)$ is

$$
=\left[\prod_{\left\{i: t_{i} \in\left(x_{j}, x_{j+1}\right)\right\}} \lambda_{j+1}\left(t_{i} \mid x_{j}\right)\right] \cdot e^{-\int_{x_{j}}^{x_{j+1}} \lambda_{j+1}\left(t \mid x_{j}\right) d t} .
$$

Since the overhaul epochs are prescheduled, the failure times in disjoint epoch periods are independent, and therefore, the joint pdf of the failure times $\left(t_{1}, t_{2}, \ldots, t_{n}\right)$ is simply the product form of $(9 b)$, that is,

$$
\begin{aligned}
f\left(t_{1}, t_{2}, \ldots, t_{n}\right)= & L(\text { data } \mid \eta, \theta, \rho) \\
= & {\left[\prod_{j=0}^{k} \prod_{t_{i} \in\left(x_{j}, x_{j+1}\right)} \lambda_{j+1}\left(t_{i} \mid x_{j}\right)\right] } \\
& \cdot e^{-\sum_{j=0}^{k} \int_{x_{j}}^{x_{j+1}} \lambda_{j+1}\left(t \mid x_{j}\right) d t} \\
= & \eta^{n}\left[\prod_{j=0}^{k} \prod_{t_{i} \in\left(x_{j}, x_{j+1}\right)}\left(\frac{t_{i}-\rho x_{j}}{t_{i}-\rho x_{j}+\theta}\right)\right] \\
& \cdot e^{-\eta \sum_{j=0}^{k}\left[\left(x_{j+1}-x_{j}\right)-\theta \log \left(\left(x_{j+1}-\rho x_{j}+\theta\right) /\left(x_{j}-\rho x_{j}+\theta\right)\right)\right]},
\end{aligned}
$$

where $x_{k+1}=T$.

\section{Bayesian Procedure}

5.1. Informative Prior. Suppose that the analyst is able to anticipate the following:

(a) the asymptotic value $\lambda_{\infty} \equiv \eta$ of the intensity function,

(b) the time $t_{r}$ at which the failure intensity is $r$ times the asymptotic value $(r<1)$, that is,

$$
\begin{aligned}
& \frac{\lambda\left(t_{r}\right)}{\lambda_{\infty}}=r ; \quad x_{i}<t_{r}<x_{i+1} \\
& \Longrightarrow t_{r}=\left(\frac{(1-r) \rho x_{i}+r \theta}{1-r}\right) .
\end{aligned}
$$

Now, we assume a Gamma density for $\eta$ and $t_{r}$, both for the mathematical tractability and flexibility. Therefore,

$$
\begin{aligned}
& g(\eta)=\frac{b^{a}}{\Gamma(a)} \eta^{a-1} \exp (-b \eta), \quad a, b>0, \\
& g\left(t_{r}\right)=\frac{d^{c}}{\Gamma(c)}\left(t_{r}\right)^{c-1} \exp \left(-d t_{r}\right), \quad c, d>0,
\end{aligned}
$$

where the gamma parameters are related to the prior mean and standard deviation by

$$
\begin{array}{ll}
a=\left(\frac{\mu_{\eta}}{\sigma_{\eta}}\right)^{2}, & b=\frac{\mu_{\eta}}{\sigma_{\eta}^{2}}, \\
c=\left(\frac{\mu_{t}}{\sigma_{t}}\right)^{2}, & d=\frac{\mu_{t}}{\sigma_{t}^{2}} .
\end{array}
$$

The analyst can use the fact that a $\rho$ value closer to 0 (to 1 ) indicates less (more) effectiveness of the overhaul actions to formulate an informative prior on $\rho$. We assume that the analyst is able to anticipate both a prior mean and a standard deviation on $\rho$, say $\mu_{\rho}$ and $\sigma_{\rho}$, then we formalize this information through a beta density

$$
g(\rho)=\frac{\rho^{p-1}(1-\rho)^{q-1}}{\beta(p, q)}, \quad 0 \leq \rho \leq 1,
$$

where the values of the parameters related to the prior mean and standard deviation are

$$
\begin{aligned}
& p=\frac{\left(1-\mu_{\rho}\right) \mu_{\rho}^{2}}{\sigma_{\rho}^{2}}-\mu_{\rho}, \\
& q=p \frac{\left(1-\mu_{\rho}\right)}{\mu_{\rho}} .
\end{aligned}
$$

5.2. Joint Prior Density. Assuming the prior independence of the parameters $(\eta, \theta, \rho)$, and using (11) to find density of $\theta$, the joint prior density $g(\eta, \theta, \rho)$ is given by

$$
\begin{aligned}
g(\eta, \theta, \rho) \propto & \eta^{a-1} e^{-b \eta}\left(\frac{(1-r) \rho x_{i}+r \theta}{1-r}\right)^{c-1} \\
& \times e^{-d\left(\left((1-r) \rho x_{i}+r \theta\right) /(1-r)\right)} \rho^{p-1}(1-\rho)^{q-1} .
\end{aligned}
$$

5.3. Joint Posterior Density on Model Parameters. By combining the likelihood (10) with the joint prior density (16), the joint posterior density on the model parameters is given by

$$
\begin{aligned}
\pi(\eta, \theta, \rho \mid \text { data })= & \frac{1}{D} \eta^{n} \cdot\left[\prod_{j=0}^{k} \prod_{t_{i} \in\left(x_{j}, x_{j+1}\right)}\left(\frac{t_{i}-\rho x_{j}}{t_{i}-\rho x_{j}+\theta}\right)\right] \\
& \cdot e^{-\eta \sum_{j=0}^{k}\left[\left(x_{j+1}-x_{j}\right)-\theta \log \left(\left(x_{j+1}-\rho x_{j}+\theta\right) /\left(x_{j}-\rho x_{j}+\theta\right)\right)\right]} \\
& \cdot \eta^{a-1} \cdot e^{-b \eta} \cdot\left(\frac{(1-r) \rho x_{i}+r \theta}{1-r}\right)^{c-1} \\
& \cdot e^{\left.-d\left(\left((1-r) \rho x_{i}+r \theta\right) /(1-r)\right)\right)} \\
& \cdot \rho^{p-1} \cdot(1-\rho)^{q-1}
\end{aligned}
$$

where 


$$
\begin{aligned}
D & =\Gamma(n+a) \int_{0}^{\infty} \int_{0}^{1} \frac{f(\theta, \rho \mid \text { data })}{\left(b+\sum_{j=0}^{k}\left[\left(x_{j+1}-x_{j}\right)-\theta \log \left(\left(x_{j+1}-\rho x_{j}+\theta\right) /\left(x_{j}-\rho x_{j}+\theta\right)\right)\right]\right)^{n+a}} d \rho d \theta, \\
f(\theta, \rho \mid \text { data }) & =\left[\prod_{j=0}^{k} \prod_{t_{i} \in\left(x_{j}, x_{j+1}\right)}\left(\frac{t_{i}-\rho x_{j}}{t_{i}-\rho x_{j}+\theta}\right)\right] \cdot\left(\frac{(1-r) \rho x_{i}+r \theta}{1-r}\right)^{c-1} \cdot e^{-d\left(\left((1-r) \rho x_{i}+r \theta\right) /(1-r)\right)} \cdot \rho^{p-1} \cdot(1-\rho)^{q-1} .
\end{aligned}
$$

\section{Posterior Inference on the Expected Number of Failures in a Future Time Interval}

Let $M_{\tau}$ be the expected number of failures in the future time interval $(T, \tau)$,

$$
\begin{aligned}
M(\tau) & =E\{N(T, \tau)\} \\
& =\eta\left[(\tau-T)-\theta \log \left(\frac{\tau-\rho s+\theta}{T-\rho s+\theta}\right)\right],
\end{aligned}
$$

where

$$
s= \begin{cases}T & \text { if overhaul is performed at } T, \\ x_{k}, & \text { otherwise }\end{cases}
$$

If no overhaul is performed after $T$, the conditional distribution of $M_{\tau}$ is Poisson with mean $M(\tau)$ equal to the expected number of failures in the time interval $(T, \tau)$,

$$
\begin{aligned}
\operatorname{Pr}\left\{M_{\tau}=m \mid \eta, \theta, \rho ; \text { data }\right\}= & \frac{[M(\tau)]^{m} \exp [-M(\tau)]}{m !}, \\
\operatorname{Pr}\left\{M_{\tau}=m \mid \text { data }\right\}= & \int_{0}^{\infty} \int_{0}^{\infty} \int_{0}^{1} \pi(\eta, \theta, \rho) \cdot \frac{[M(\tau)]^{m} \exp [-M(\tau)]}{m !} d \rho d \theta d \eta \\
= & \frac{1}{D} \cdot \frac{\Gamma(n+a+m)}{m !} \int_{0}^{\infty} \int_{0}^{1} f(\theta, \rho \mid \text { data }) \\
& \cdot \frac{\left[X_{\tau}^{(\theta)}\right]^{m}}{\left(b+\sum_{j=0}^{k}\left[\left(x_{j+1}-x_{j}\right)-\theta \log \left(\left(x_{j+1}-\rho x_{j}+\theta\right) /\left(x_{j}-\rho x_{j}+\theta\right)\right)\right]+X_{\tau}^{(\theta)}\right)^{n+a+m}} d \rho d \theta,
\end{aligned}
$$

where

$$
\begin{aligned}
X_{\tau}^{(\theta)}= & (\tau-T) \\
& -\theta \log \left(\frac{\tau-\rho s+\theta}{T-\rho s+\theta}\right) .
\end{aligned}
$$

The posterior mean of $M_{\tau}$ is given by the posterior expecta- tion of $M(\tau)$,

$$
E\left\{M_{\tau} \mid \text { data }\right\}=\sum_{m=0}^{\infty} m \cdot \operatorname{Pr}\left\{M_{\tau}=m \mid \text { data }\right\} .
$$

After the transformation of variables, we have the following:

$$
E\left\{M_{\tau} \mid \text { data }\right\}=\frac{(n+a) !}{D} \int_{0}^{\infty} \int_{0}^{1} f(\theta, \rho \mid \text { data }) \cdot \frac{X_{\tau}^{(\theta)}}{\left(b+\sum_{j=0}^{k}\left[\left(x_{j+1}-x_{j}\right)-\theta \log \left(\left(x_{j+1}-\rho x_{j}+\theta\right) /\left(x_{j}-\rho x_{j}+\theta\right)\right)\right]\right)^{n+a+1}} d \rho d \theta
$$

A conservative $\gamma$ Bayes upper credibility limit for $M_{\tau}$ is obtained by finding the smallest value of which $m_{u}$ satisfies

$$
\operatorname{Pr}\left\{M_{\tau} \leq m_{u} \mid \text { data }\right\} \geq \gamma .
$$

\section{Posterior Inference on the Future Failure Times}

Given the observed data, we are interested in predicting the $m$ th future failure time $t_{n+m}$, under the assumption thatno 
overhaul is performed after $T$,

$$
\begin{aligned}
M_{m} & =E\left\{N\left(T, t_{n+m}\right)\right\} \\
& =\eta\left[\left(t_{n+m}-T\right)-\theta \log \left(\frac{t_{n+m}-\rho s+\theta}{T-\rho s+\theta}\right)\right],
\end{aligned}
$$

where

$$
s= \begin{cases}T & \text { if overhaul is performed at } T \\ x_{k}, & \text { otherwise }\end{cases}
$$

$M_{m}$ is gamma distributed with shape parameter $\mathrm{m}$ and unit scale parameter. Thus, the conditional pdf of $t_{n+m}$, with
$t_{n+m}>T$, is given by

$$
\begin{aligned}
p\left(t_{n+m} \mid \eta, \theta, \rho ; \text { data }\right)= & \frac{1}{\Gamma m} \eta^{m}\left[X_{m}^{(\theta)}\right]^{m-1} \exp \left[-\eta X_{m}^{(\theta)}\right] \\
& \times\left[\frac{t_{n+m}-\rho s}{t_{n+m}-\rho s+\theta}\right],
\end{aligned}
$$

where

$$
X_{m}^{(\theta)}=\left(t_{n+m}-T\right)-\theta \log \left(\frac{t_{n+m}-\rho s+\theta}{T-\rho s+\theta}\right) .
$$

The predictive density function of $t_{n+m}$ is given by

$$
\begin{aligned}
h\left\{t_{n+m} \mid \text { data }\right\}= & \int_{0}^{\infty} \int_{0}^{\infty} \int_{0}^{1} \pi(\eta, \theta, \rho) \cdot p\left(t_{n+m} \mid \eta, \theta, \rho \text {; data }\right) d \rho d \theta d \eta \\
= & \frac{1}{D} \cdot \frac{\Gamma(n+a+m)}{\Gamma(m)} \\
& \times \int_{0}^{\infty} \int_{0}^{1} f(\theta, \rho \mid \text { data }) \cdot \frac{\left[X_{m}^{(\theta)}\right]^{m-1} \cdot\left[\left(t_{n+m}-\rho s\right) /\left(t_{n+m}-\rho s+\theta\right)\right]}{\left(b+\sum_{j=0}^{k}\left[\left(x_{j+1}-x_{j}\right)-\theta \log \left(\left(x_{j+1}-\rho x_{j}+\theta\right) /\left(x_{j}-\rho x_{j}+\theta\right)\right)\right]+X_{m}^{(\theta)}\right)^{n+a+m} d \rho d \theta .}
\end{aligned}
$$

A Bayes prediction interval of a given probability content $\gamma$ is defined as the interval $I$ such that

$$
\operatorname{Pr}\{I \mid \text { data }\}=\int_{I} h\left(t_{n+m} \mid \text { data }\right) d t_{n+m}+\gamma
$$

\section{Sensitivity Analysis}

Sensitivity analysis is carried out with respect to the prior information on $\rho$, namely the prior mean $\mu_{\rho}$ and the standard deviation $\sigma_{\rho}$, by evaluating $M_{\tau}$ : expected number of failures in the future time interval $(T, \tau)$ when overhaul is performed at $T$ and not performed at $T$, over a reasonable range of values for $\mu_{\rho}$ and $\sigma_{\rho}$. If the slight change in values of $\mu_{\rho}$ and $\sigma_{\rho}$ does not have much effect on $M_{\tau}$ then the proposed prior is robust.

\section{Numerical Application}

Consider the following hypothetical data for illustrative purpose.

The failure times $(n=18)$ and overhaul epochs are given in Table 1, major overhauls marked with $*$. We have assumed it to be a time-truncated sample with failures observed for 1500 units. Four major overhauls are assumed to be performed at times different from failure times.

The proportional age reduction 2-EBP model is adequate for this hypothetical data set in contrast to pre-existing proportional age reduction power law process model as its log-likelihood value obtained using (10) is -95.4606 which is greater than the log-likelihood value -96.1537 of power law process model.

Suppose that analyst is able to anticipate a prior mean $\mu_{\eta}=0.133$ and $\sigma_{\eta}=0.004(a=4, b=30)$.

In addition, from previous experiences, the analyst possesses a vague belief that the failure intensity, at the time $t_{r}=753$ units, is nearly half its asymptotic value: $\lambda(753) / \lambda_{\infty}=0.5$. Then, he formalizes his prior knowledge on $t_{r}$ through the exponential density having mean $\mu_{t}=753$, so that $c=1$ and $d=0.001$. As $x_{i}<t_{r}<x_{i+1}$, therefore $600<753<900$. Hence, $x_{i}=600$.

Again, the analyst possesses a vague belief that the overhaul actions are quite effective, and then he chooses the beta density for the improvement parameter with prior mean $\mu_{\rho}=0.6$ and standard deviation $\sigma_{\rho}=0.26(p=1.5$ and $q=$ $1)$.

Now, suppose that the above failure process is time truncated at $T=1200$ units. On the basis of the observed data, we predict the future failure times under two hypotheses:

(a) major overhaul is performed at $T$,

(b) no overhaul is performed at $T$.

All further repair actions are assumed to be minimal repairs. The remaining observed values, that is, $\{1230,1268,1330$, and 1447$\}$ have been used for comparison with the predicted values.

In Table 2, we compare the occurred failure times $t_{13+m}(m=1,2,3,4,5)$ with the Bayes prediction of the $m$ th future failure times under both the above hypotheses; in particular, the lower $\left(t_{l}\right)$ and upper $\left(t_{u}\right)$ limits of the 
TABle 1: Failure times and overhaul epochs.

\begin{tabular}{lcccccccccc}
\hline 202 & 265 & $300^{*}$ & 363 & 508 & 571 & $600^{*}$ & 755 & 770 & 818 & 868 \\
\hline $900^{*}$ & 999 & 1054 & 1068 & 1108 & $1200^{*}$ & 1230 & 1268 & 1330 & 1376 & 1447 \\
\hline
\end{tabular}

TABLE 2: Comparison of the occurred failure times $t_{13+m}(m=1,2,3,4,5)$ with the Bayes prediction under the hypothesis that overhaul is or is not performed at $T$.

\begin{tabular}{|c|c|c|c|c|c|}
\hline \multirow[b]{2}{*}{$m$} & \multirow{2}{*}{$\begin{array}{c}\text { Actual } \\
t_{13+m}\end{array}$} & \multicolumn{2}{|c|}{ Overhaul at $T$} & \multicolumn{2}{|c|}{ No overhaul at $T$} \\
\hline & & $t_{l}$ & $t_{u}$ & $t_{l}$ & $t_{u}$ \\
\hline 1 & 1230 & 1204.3 & 1421.0 & 1202.4 & 1342.0 \\
\hline 2 & 1268 & 1226.1 & 1514.0 & 1216.4 & 1420.0 \\
\hline 3 & 1330 & 1255.2 & 1588.4 & 1236.6 & 1486.0 \\
\hline 4 & 1376 & 1286.5 & 1653.6 & 1259.5 & 1546.8 \\
\hline 5 & 1447 & 1318.4 & 1713.5 & 1283.5 & 1603.7 \\
\hline
\end{tabular}

TABLE 3: Comparison of occurred failure times with bayes prediction.

\begin{tabular}{lccccc}
\hline$n$ & $T$ & Actual $t_{n+1}$ & $90 \%$ Lower Limit & Median Value & $90 \%$ Upper Limit \\
\hline 13 & $x_{4}=1200$ & 1230 & 1204.3 & 1258.1 & 1421.0 \\
14 & $t_{14}=1230$ & 1268 & 1233.4 & 1275.7 & 1417.0 \\
15 & $t_{15}=1268$ & 1330 & 1271.0 & 1308.0 & 1435.2 \\
16 & $t_{16}=1330$ & 1376 & 1332.7 & 1366.1 & 1482.0 \\
17 & $t_{17}=1376$ & 1447 & 1378.5 & 1409.4 & 1518.1 \\
\hline
\end{tabular}

TABle 4: Posterior mean and 0.95 upper credibility limit of the number of failures in the future time interval $(1200,1500)$.

\begin{tabular}{|c|c|c|c|}
\hline & \multicolumn{2}{|c|}{ Posterior Mean } & $\begin{array}{l}0.95 \text { Upper credibility } \\
\text { limit }\end{array}$ \\
\hline \multicolumn{2}{|l|}{ Overhaul at $T$} & 5.327 & 11 \\
\hline \multicolumn{2}{|l|}{ No Overhaul at $T$} & 7.612 & 14 \\
\hline \multicolumn{4}{|c|}{ TABLE 5: Sensitivity analysis with respect to the prior mean of $\rho$. } \\
\hline $\begin{array}{l}\text { Percentage } \\
\text { Deviation }\end{array}$ & $\mu_{\rho}$ & $\begin{array}{c}M_{\tau} \text { with no } \\
\text { overhaul at } T\end{array}$ & $\begin{array}{c}M_{\tau} \text { with overhaul } \\
\text { at } T\end{array}$ \\
\hline$-1 \%$ & 0.594 & 7.599 & 5.359 \\
\hline$+1 \%$ & 0.606 & 7.618 & 5.312 \\
\hline$-2 \%$ & 0.588 & 7.588 & 5.386 \\
\hline$+2 \%$ & 0.612 & 7.628 & 5.287 \\
\hline$-3 \%$ & 0.582 & 7.578 & 5.410 \\
\hline$+3 \%$ & 0.618 & 7.639 & 5.261 \\
\hline
\end{tabular}

0.90 Bayes equal-tail credibility interval are shown. It clearly shows the advantages of performing overhauls as the future failure times are delayed considerably by overhaul.

In Table 3, we compare the observed values of $t_{n+1}(n=$ $13,14,15,16,17)$ with the lower and upper limits of the Bayes 0.90 equal-tail credibility interval and with the posterior median obtained using (30) and (31). This allows the analyst to assess the adequacy of the selected model and the correctness of prior information. Table 3 shows that all occurred failures fall well within the Bayes prediction interval and are quite close to the respective posterior median, thus
TABLE 6: Sensitivity analysis with respect to the standard deviation of $\rho$.

\begin{tabular}{lccc}
\hline $\begin{array}{l}\text { Percentage } \\
\text { deviation }\end{array}$ & $\sigma_{\rho}$ & $\begin{array}{c}M_{\tau} \text { with no } \\
\text { overhaul at } T\end{array}$ & $\begin{array}{c}M_{\tau} \text { with overhaul } \\
\text { at } T\end{array}$ \\
\hline$-1 \%$ & 0.2574 & 7.610 & 5.352 \\
$+1 \%$ & 0.2626 & 7.614 & 5.322 \\
$-2 \%$ & 0.2548 & 7.596 & 5.367 \\
$+2 \%$ & 0.2652 & 7.619 & 5.308 \\
$-3 \%$ & 0.2522 & 7.591 & 5.381 \\
$+3 \%$ & 0.2678 & 7.625 & 5.292 \\
\hline
\end{tabular}

indicating the adequacy of the 2-EBP to model the given data set.

Table 4 clearly shows that the, 95\% upper credibility limit for the number of failures in future time interval $(1200,1500)$ when major overhaul is performed at $T=1200$ is equal to 11 failures and the posterior mean $=5.327$ failures, very close to the number of failures, namely 5, actually occurred in (1200, 1500).

On the contrary in the case of no overhaul 95\% upper credibility limit for the number of failures in future time interval $(1200,1500)$ is equal to 14 failures which is more than that in major overhaul case, and the posterior mean is 7.612 which is again more than the value obtained in the major overhaul case. These results show the considerable advantages arising from performing a major overhaul at $T$.

Tables 5 and 6 show that the change in values of $\mu_{\rho}$ and $\sigma_{\rho}$ does not have much effect on $M_{\tau}$, so the proposed prior is robust. 


\section{Conclusion}

In this paper, the prediction of future failures of a deteriorating repairable mechanical system subject to minimal repairs and periodic overhauls has been done using Bayesian approach. The effect of overhauls on the reliability of the system has been modeled using a proportional age reduction model, and the failure process between two successive overhauls has been modeled using 2-parameter EngelhardtBain process (2-EBP). The prediction of the future failure times and the number of failures in a future time interval has been done on the basis of the observed data and of a number of suitable prior densities reflecting varied degrees of belief on the failure/repair process and effectiveness of overhauls. The advantages of overhauls have been highlighted using a numerical application and sensitivity analysis of the improvement parameter carried out.

\section{Nomenclature}

\begin{tabular}{|c|c|}
\hline$n:$ & Total no. of failures \\
\hline$k:$ & Total no. of overhauls \\
\hline$x_{i}:$ & $i$ th overhaul epoch, $i=1 \cdots k$ \\
\hline$\eta, \theta:$ & Parameters of 2 -EBP \\
\hline$\rho:$ & Improvement factor \\
\hline$t_{r}:$ & $\begin{array}{l}\text { Time at which the failure } \\
\text { intensity is } r \text { times the } \\
\text { asymptotic value }\end{array}$ \\
\hline$\lambda_{1}(t):$ & Initial failure intensity \\
\hline$\lambda_{j+1}\left(t \mid x_{j}\right):$ & $\begin{array}{l}\text { Conditional intensity function } \\
\text { at a generic time } t \text { in the interval } \\
\left(x_{j}, x_{j+1}\right)\end{array}$ \\
\hline$E\left\{N\left(x_{j}, x_{j+1}\right)\right\}:$ & $\begin{array}{l}\text { Expected number of failures } \\
\text { between two successive overhaul } \\
\text { epochs }\end{array}$ \\
\hline$g(\eta):$ & Prior pdf on $\eta$ \\
\hline$g\left(t_{r}\right):$ & Prior pdf on $t_{r}$ \\
\hline$g(\rho):$ & Prior pdf on $\rho$ \\
\hline$a, b:$ & Prior gamma parameters of $\eta$ \\
\hline$c, d:$ & Prior gamma parameters of $t_{r}$ \\
\hline$p, q:$ & Prior beta parameters of $\rho$ \\
\hline$\mu_{\eta}, \sigma_{\eta}^{2}:$ & Prior mean and variance of $\eta$ \\
\hline$\mu_{t_{r}}, \sigma_{t_{r}}^{2}:$ & Prior mean and variance of $t_{r}$ \\
\hline$\mu_{\rho}, \sigma_{\rho}^{2}:$ & Prior mean and variance of $\rho$ \\
\hline$g\left(\eta, t_{r}, \rho\right):$ & Joint prior pdf of $\eta, t_{r}, \rho$ \\
\hline$\pi(\eta, \theta, \rho \mid$ data $):$ & Joint posterior pdf of $\eta, \theta, \rho$ \\
\hline$M_{\tau}:$ & $\begin{array}{l}\text { Expected number of failures in } \\
\text { the future time interval }(T, \tau)\end{array}$ \\
\hline$t_{n+m}:$ & $m$ th future failure time \\
\hline NHPP: & $\begin{array}{l}\text { Nonhomogeneous poisson } \\
\text { process }\end{array}$ \\
\hline 2-EBP: & $\begin{array}{l}\text { 2-parameter Engelhardt-Bain } \\
\text { process }\end{array}$ \\
\hline PLP: & Power law process. \\
\hline
\end{tabular}

\section{Acknowledgment}

The authors are grateful to the referees for their valuable comments and suggestions that improved this paper by making it more informative.

\section{References}

[1] H. Pham and H. Wang, "Imperfect maintenance," European Journal of Operational Research, vol. 94, no. 3, pp. 425-438, 1996.

[2] M. A. K. Malik, "Reliable preventive maintenance scheduling," AIIE Transactions, vol. 11, no. 3, pp. 221-228, 1979.

[3] I. Shin, T. J. Lim, and C. H. Lie, "Estimating parameters of intensity function and maintenance effect for repairable unit," Reliability Engineering and System Safety, vol. 54, no. 1, pp. 110, 1996.

[4] L. J. Bain and M. Engelhardt, "Inferences on the parameters and current system reliability for a time truncated weibull process," Technometrics, vol. 22, no. 3, pp. 421-426, 1980.

[5] L. J. Bain and M. Engelhardt, "Prediction intervals for the Weibull Process," Technometrics, vol. 20, pp. 167-169, 1978.

[6] L. H. Crow, "Reliability analysis for complex, repairable systems," Reliability and Biometry, vol. 13, no. 6, pp. 379-410, 1974.

[7] M. Guida, R. Calabria, and G. Pulcini, "Bayes inference for a non-homogeneous Poisson process with power intensity law," IEEE Transactions on Reliability, vol. 38, no. 5, pp. 603-609, 1989.

[8] L. Lee and S. K. Lee, "Some results on inference for the weibull process," Technometrics, vol. 20, no. 1, pp. 41-45, 1978.

[9] S. E. Rigdon and A. P. Basu, Statistical Methods for the Reliability of Repairable Systems, John Wiley, New York, NY, USA, 2000.

[10] A. Sen, "Bayesian estimation and prediction of the intensity of the power law process," Journal of Statistical Computation and Simulation, vol. 72, no. 8, pp. 613-631, 2002.

[11] J. W. Yu, G. L. Tian, and M. L. Tang, "Predictive analyses for nonhomogeneous Poisson processes with power law using Bayesian approach," Computational Statistics and Data Analysis, vol. 51, no. 9, pp. 4254-4268, 2007.

[12] D. R. Cox and P. A. W. Lewis, The Statistical Analysis of Series of Events, 1966.

[13] N. Jack, "Analysing event data from a repairable machine subject to imperfect preventive maintenance," Quality and Reliability Engineering International, vol. 13, no. 4, pp. 183186, 1997.

[14] G. Pulcini, "On the overhaul effect for repairable mechanical units: a Bayes approach," Reliability Engineering and System Safety, vol. 70, no. 1, pp. 85-94, 2000.

[15] G. Pulcini, "On the prediction of future failures for a repairable equipment subject to overhauls," Communications in Statistics, vol. 30, no. 4, pp. 691-706, 2001.

[16] M. Engelhardt and L. J. Bain, "On the mean time between failures for repairable systems," IEEE Transactions on Reliability, vol. 35, no. 4, pp. 419-422, 1986.

[17] G. Pulcini, "A bounded intensity process for the reliability of repairable equipment," Journal of Quality Technology, vol. 33, no. 4, pp. 480-492, 2001.

[18] L. Attardi and G. Pulcini, "A new model for repairable systems with bounded failure intensity," IEEE Transactions on Reliability, vol. 54, no. 4, pp. 572-582, 2005. 

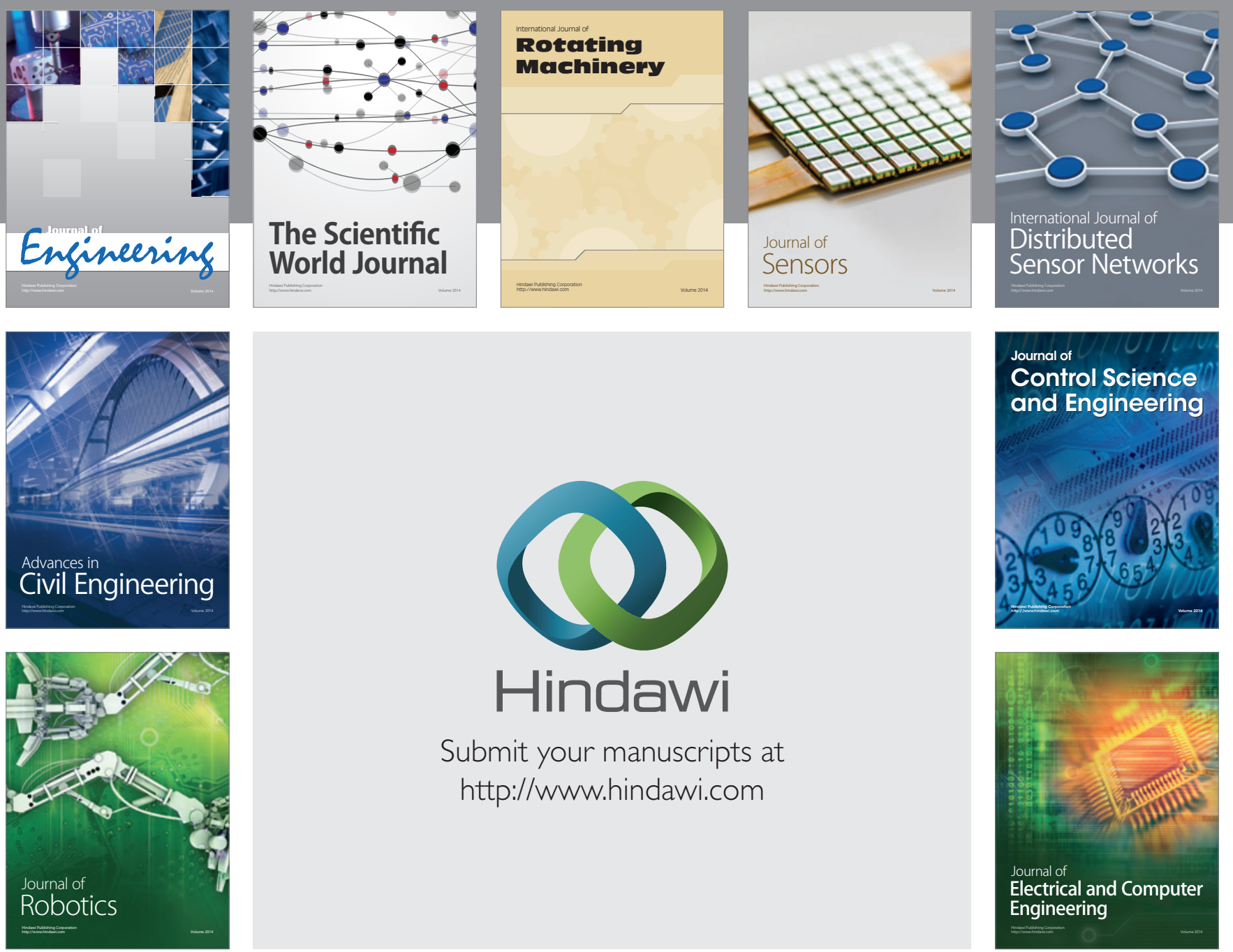

Submit your manuscripts at

http://www.hindawi.com
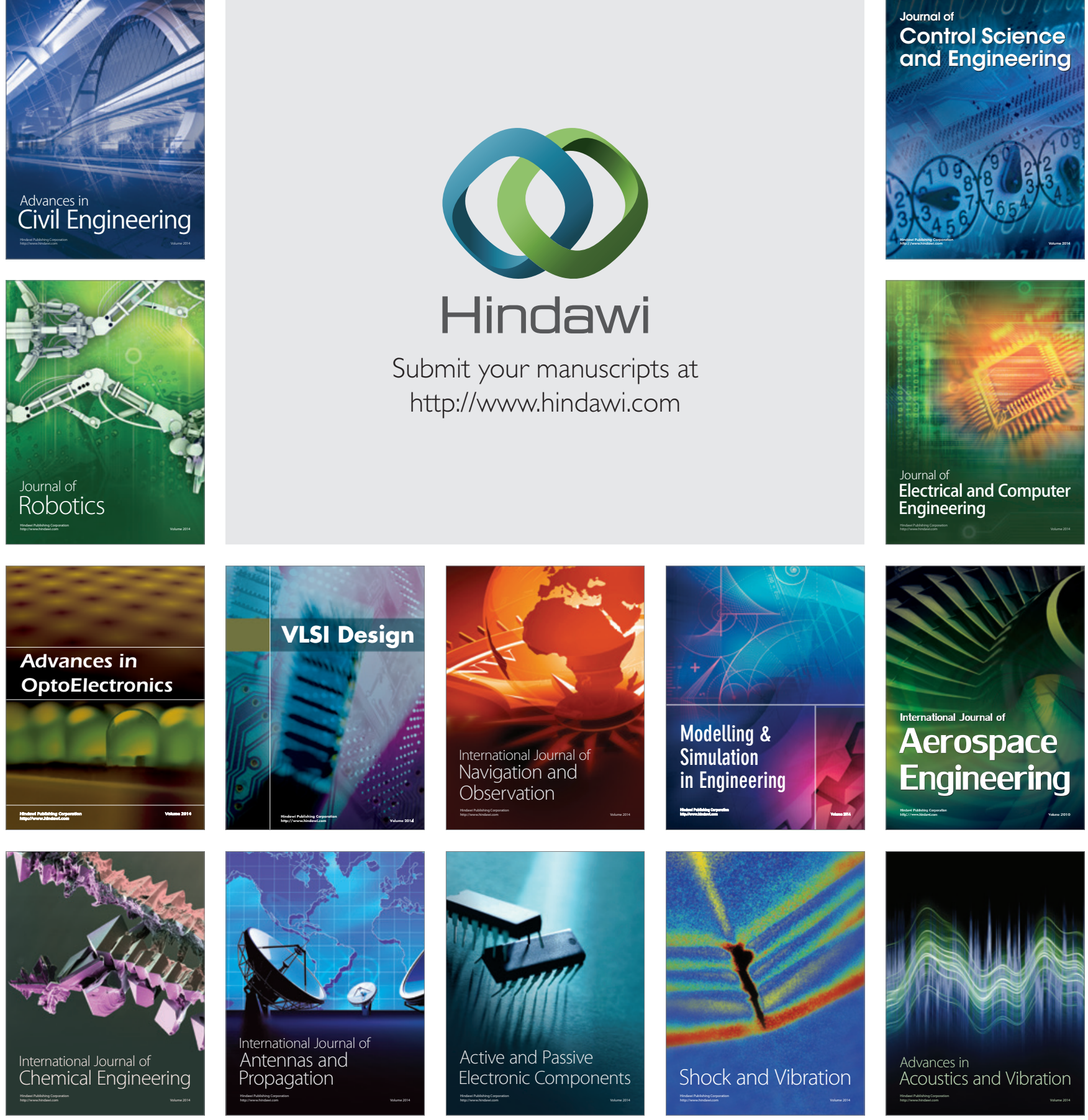